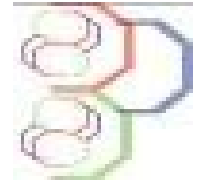

Journal of Applied Biosciences 145: 14974 - 14984

ISSN 1997-5902

\title{
Etudes comparatives de la composition chimique et de l'activité coagulante des graines de Cucumeropsis mannii Naud., Arachis hypogaea L. et Moringa oleifera Lam. dans la clarification des eaux de surface.
}

\author{
Ntalani Hermeline ${ }^{1,3 *}$, Ngouanou Ravelle Duclérine1, Makomo Hubert1, Elouma Ndinga Arnold \\ Murphy1, Boungou-Tsona Ghislaine2,3, Loumouamou Aubin Nestor ${ }^{2,3}$, Mouloungui Zéphirin ${ }^{4}$, \\ Ouamba Jean-Maurille ${ }^{1}$ \\ 1. Unité de Chimie du Végétal et de la Vie, Faculté des Sciences et Techniques, Université Marien NGOUABI, BP \\ 69, Brazzaville, Congo \\ 2. Equipe Pluridisciplinaire de Recherche en Alimentation et en Nutrition, Faculté des Sciences et Techniques, \\ Université Marien NGOUABI, BP 69, Brazzaville, Congo \\ 3. Institut de Recherche en Sciences Exactes et Naturelles, BP 2400, Brazzaville, Congo \\ 4. Laboratoire de Chimie Agro-Industrielle, INRA-ENSIACET, BP 44362, Toulouse, France \\ ${ }^{*}$ Auteur correspondant : tabunahermeline@gmail.com
}

Original submitted in on $5^{\text {th }}$ November 2019. Published online at www.m.elewa.org/journals/ on $31^{\text {st }}$ January 2020

https://doi.org/10.35759/JABs.v145.12

\section{RESUME}

Objectif: Cette étude a pour objectif de comparer la teneur en protéines brutes et en acides aminés des graines de $C$. mannii et de $A$. hypogaea avec celles des graines de $M$. oleifera, qui ont une activité coagulante pour la clarification de l'eau, puis d'évaluer l'activité coagulante des graines de ces deux plantes.

Méthodologie et résultats : Les teneurs en protéines brutes et en acides aminés ont été déterminées par des méthodes classiques. L'étude de l'activité coagulante a été effectuée par Jar-test sur des échantillons des eaux brutes de turbidité 128,6 NTU, 94 NTU et 89,46 NTU, prélevés dans la rivière Djoué. L'arginine, avec des teneurs de $5510 \mathrm{mg} / 100 \mathrm{~g}, 3550 \mathrm{mg} / 100 \mathrm{~g}, 4850 \mathrm{mg} / 100 \mathrm{~g}$, et l'acide glutamique, avec des teneurs de $6830 \mathrm{mg} / 100 \mathrm{~g}, 6300 \mathrm{mg} / 100 \mathrm{~g}, 6840 \mathrm{mg} / 100 \mathrm{~g}$ sont les plus abondants dans les trois plantes. Les résultats de l'étude comparative de l'activité coagulante menée sur l'échantillon d'eau brute de turbidité $94 \mathrm{NTU}$, indiquent les doses optimales respectives de $16 \mathrm{mg} / \mathrm{L}, 1400 \mathrm{mg} / \mathrm{L}, 7000 \mathrm{mg} / \mathrm{L}$ et $9000 \mathrm{mg} / \mathrm{L}$, pour les solutions de sulfate d'aluminium, $M$. oleifera, $A$. hypogaea et $C$. mannii.

Conclusion et application des résultats: Les résultats de cette étude mettent en évidence l'activité coagulante des graines de C. mannii et de A. hypogaea. Les graines de ces deux plantes peuvent donc être utilisées au niveau des ménages pour la clarification des eaux de surface dans les régions rurales des pays en développement. L'eau clarifiée pourra ensuite subir une désinfection par des méthodes physiques telles que l'ébullition et la désinfection solaire.

Mots clés: Cucumeropsis mannii, Arachis hypogaea, Moringa oleifera, coagulant, clarification, eaux 
Comparative studies of the chemical composition and the coagulanting activity of the seeds of Cucumeropsis mannii Naud., Arachis hypogaea L. and Moringa oleifera Lam. in the clarification of surface waters.

\begin{abstract}
Objective: The objective of this study is to compare the crude protein and amino acid content of $C$. mannii and $A$. hypogaea seeds with those of $M$. oleifera seeds, that have a coagulanting activity for water clarification, and to evaluate the coagulating activity seeds of these two plants.

Methodology and results: The crude protein and amino acid contents were determined by standard methods. The study of the coagulating activity has been carried out by Jar-test on samples raw waters with turbidity 128.6 NTU, 94 NTU and 89.46 NTU, taken from the Djoué River. Arginine, with contents of 5510 $\mathrm{mg} / 100 \mathrm{~g}, 3550 \mathrm{mg} / 100 \mathrm{~g}, 4850 \mathrm{mg} / 100 \mathrm{~g}$, and glutamic acid, with contents of $6830 \mathrm{mg} / 100 \mathrm{~g}, 6300$ $\mathrm{mg} / 100 \mathrm{~g}, 6840 \mathrm{mg} / 100 \mathrm{~g}$ are the most abundant in all three plants. The results of the comparative study of the coagulant activity carried out on the 94 NTU raw water turbidity sample, indicate the optimal doses of $16 \mathrm{mg} / \mathrm{L}, 1400 \mathrm{mg} / \mathrm{L}, 7000 \mathrm{mg} / \mathrm{L}$ and $9000 \mathrm{mg} / \mathrm{L}$, for aluminum sulphate solutions, M. oleifera, A. hypogaea and C. mannii .

Conclusion and application of the results: The results of this study highlight the coagulating activity of the seeds of $C$. mannii and $A$. hypogaea. The seeds of these two plants can therefore be used at the household level for the clarification of surface waters in rural areas of developing countries. The clarified water can then be disinfected by physical methods such as boiling and solar disinfection.
\end{abstract}

Key words: Cucumeropsis mannii, Arachis hypogaea, Moringa oleifera, coagulant, clarification, waters

\section{INTRODUCTION}

L'eau est une ressource naturelle indispensable à la vie. Malgré son importance, elle n'est pas disponible en tout lieu. Selon un rapport de l'OMS, près de 2,1 milliards de personnes étaient sans accès à l'eau potable dans le monde en 2017 (OMS, 2017). Plus de 40\% des habitants de la planète n'ayant pas accès à l'eau potable vivent en Afrique subsaharienne; et dans les régions rurales des pays en développement, $97 \%$ de la population n'a pas accès à un approvisionnement en eau par canalisation (OMS, 2012). Ces populations utilisent parfois des eaux de surface pouvant provenir des rivières, des ruisseaux et des mares d'eaux, pour les tâches ménagères (Linangelo, 2018; Ofouémé, 2010). Les Objectifs du Développement Durable, imposent de disposer de l'eau saine et potable (PNUD, 2019). Pour les régions rurales des pays en développement, la recherche sur les techniques endogènes de traitement des eaux à usage domestique est une alternative pour le problème de l'eau. Parmi les techniques endogènes existantes, figure la clarification des eaux de surface par les graines de M. oleifera (Kaboré, 2013 ; Sotheeswaran, 2011 ; Fatombi, 2007), une plante dont l'activité coagulante a été démontrée par plusieurs auteurs (Kweku, 2009, Nwaiwu, 2011). La coagulation est une étape importante dans le traitement physico-chimique des eaux brutes. Elle consiste à neutraliser la charge négative des colloïdes indécantables, par injection et dispersion rapide d'un réactif chimique, le coagulant. Celui-ci apporte au milieu des cations multivalents libres ou liés à une macromolécule organique, qui provoquent la déstabilisation des colloïdes et favorisent ainsi leur rapprochement en vue de leur agglomération (Lugube, 2015). Le coagulant peut être d'origine minérale: Sulfate d'aluminium, Chlorure de fer III, Sulfate de fer II, ou organique: Polychlorure de diallyldiméthylammonium, Epichlorhydrinediméthylamine (Hendricks, 2006,). La coagulation est suivie de la floculation qui se fait à une vitesse lente dans le but de permettre l'agglomération des colloïdes déstabilisées, en flocons volumineux et décantables. Les procédés de coagulation/floculation permettent d'éliminer la turbidité de l'eau, mais aussi les germes microbiologiques qui peuvent se cacher dans les 
particules colloidales (Degremont, 2005). Afin de mettre à la disposition des populations des zones rurales des pays en développement, des procédés d'assainissement des eaux brutes au niveau des ménages, par la clarification des eaux de surface en utilisant des coagulants naturels, issus des plantes qui se trouvent dans leur environnement, et d'élargir la gamme des coagulants végétaux, nous nous intéressons à la recherche des produits d'origine végétal ayant une activité coagulante. Dans le cadre de ce travail, nous avons porté notre

\section{MATERIEL ET METHODES}

Matériel végétal: Le matériel végétal est constitué des graines de C. mannii, A. hypogaea et M. oleifera. La récolte a été effectuée dans la zone sud de la République du Congo, au mois de novembre de l'année 2018. Les graines de $C$. mannii et $A$. hypogaea ont été récoltées près du village Mboulankio situé à 45 kilomètres de Brazzaville et celles de $M$. oleifera, dans l'arrondissement 1 de la ville de Brazzaville. Les trois espèces végétales ont été identifiées par un botaniste et des spécimens ont été enregistrés à l'herbier de I'Institut des Recherches en Sciences Exactes et Naturelles respectivement sous les numéros 1,2 et 3 .

Etude de la composition chimique: L'étude de la composition chimique a porté sur les teneurs en lipides totaux, en protéines totales, en acides aminés, en glucides totaux et en amidon total. Le taux d'humidité et la teneur en cendres brutes ont également été déterminés. Trois traitements ont été effectués et la prise d'essai pour chaque traitement est de 100 grammes.

Teneur en lipides totaux, teneur en cendres brutes et taux d'humidité : La teneur en lipides a été obtenue par extraction au Soxhlet à l'éther de pétrole ; celle des cendres brutes après incinération de la matière organique et pesée du résidu. Pour l'humidité la prise d'essai de $100 \mathrm{~g}$ a été soumise à la dessiccation à $70^{\circ} \mathrm{C}$ et la perte de masse a été déterminée par pesée.

Teneurs en Protéines totales et en acides aminés : La teneur en protéines brutes a été obtenue à partir de la teneur en azote déterminée par la méthode Kjeldhal et celle des acides aminés selon les normes NF EN ISO 13903 et NF EN ISO 13904.

Identification des acides aminés : Les acides aminés libres ont été extraits avec de l'acide chlorhydrique dilué. Les macromolécules azotés co-extraites ont été précipitées et éliminés par filtration. Les acides aminés sont ensuite séparés par chromatographie sur choix sur les graines de $C$. mannii et de $A$. hypogaea. L'objectif de notre étude est d'évaluer l'activité coagulante des graines de $C$. mannii et de A. hypogaea afin de la comparer à celles des graines de $M$. oleifera, un coagulant naturel végétal dont l'activité coagulante est due à des polyélectrolytes cationiques de type polypeptide (Atakpama, 2014), et du sulfate d'aluminium, le coagulant minéral le plus utilisé pour le traitement des eaux destinées à la consommation humaine (Cardot, 2010).

échangeur d'ions et déterminés par réaction avec la minhydrine avec détection photométrique.

Teneurs en glucides totaux et en amidon total : Les glucides totaux ont été obtenus par calcul, selon la formule $100-[$ (humidité) + (protéine) + (lipides totaux) + cendres brutes)]. La teneur en amidon a été déterminée selon la norme NF EN ISO 10520.

Etude de l'activité coagulante: L'étude de l'activité coagulante a consisté à évaluer d'abord l'activité coagulante des graines de $C$. mannii et de $A$. hypogaea. Ensuite, nous avons mené une étude comparative de l'activité coagulante des graines de ces deux plantes avec celles des graines de M. oleifera et du sulfate d'aluminium. Cette 'étude a porté sur le dosage des ions $\mathrm{Al}^{3+}$, $\mathrm{Fe}^{3+}$ et $\mathrm{Fe}^{2+}$ dans les solutions préparées, le suivi de l'élimination de la turbidité, la variation du $\mathrm{pH}$ et de la conductivité et sur la teneur en matière organique apportée à l'eau traitée.

Description de la zone d'échantillonnage: Les échantillons d'eaux brutes de turbidité 128,6 NTU, 94 NTU et 89,46 NTU ont été prélevés en novembre 2018, dans la rivière Djoué, au sud de Brazzaville. Les coordonnées géographiques indiquent : 04 $18^{\prime} 34^{\prime \prime}$ de latitude Sud, $015^{\circ} 13^{\prime} 36^{\prime \prime}$ de longitude Est et $270 \mathrm{~m}$ d'altitude au-dessus de la mer.

Préparation des solutions : Les graines de C. mannii $A$. hypogaea et $M$. oleifera ont été décortiquées, séchées et broyées. Pour chaque plante, $100 \mathrm{~g}$ de produit obtenu ont été dispersés dans $1000 \mathrm{~mL}$ d'eau distillée. La solution de sulfate d'aluminium, a été préparée à la concentration de $10 \mathrm{~g} / \mathrm{L}$; concentration utilisée à la société nationale de traitement et de distribution de l'eau au Congo. Nous avons également préparé les solutions à $10 \mathrm{~g} / \mathrm{L}$ de chlorure de fer III et de sulfate de fer II.

Essais de clarification des échantillons d'eaux brutes de 128,60 NTU et 89,46 NTU : Les essais de 
clarification des échantillons d'eaux brutes de turbidité 128,60 NTU et 89,46 NTU ont été effectués par JarTest (Cardot, 2010). Pour la réalisation du Jar-Test, $1000 \mathrm{~mL}$ d'échantillon d'eau brute ont été introduit dans les béchers d'un floculateur Lovibond ET 740, suivi de l'ajout de différents volumes croissants des solutions de poudre des graines de C. mannii, pour l'échantillon d'eau brute de 89,46 NTU et de $A$. hypogaea, pour celui de 128,6 NTU. Après une agitation rapide de 180 tours par seconde pendant 3 minutes et une agitation lente de 18 tours par seconde pendant 20 minutes, les échantillons d'eaux traités ont été soumis à la décantation pendant 30 minutes. La température, le $\mathrm{pH}$, la turbidité et la conductivité ont été mesurés; la turbidité a encore été mesurée après une filtration des échantillons d'eaux traités et décantés. Ces paramètres physico chimiques ont également été mesurés pour les eaux brutes avant le traitement par Jar-Test. Trois traitements Jar-Test ont été effectués pour chaque échantillon d'eau brute traité avec les différentes doses des solutions de C. manniii et $A$. hypogaea

Etude comparée des essais de clarification de l'échantillon d'eau brute de 94 NTU

Dosage des ions $\mathrm{Al}^{3+}, \mathrm{Fe}^{3+}$ et $\mathrm{Fe}^{2+}$ dans les solutions préparées : Les ions $\mathrm{Al}^{3+}, \mathrm{Fe}^{3+}$ et $\mathrm{Fe}^{2+}$ ont été dosés dans les solutions préparées de C. mannii , $A$.

\section{RESULTATS ET DISCUSSION}

Etude de la composition chimique : Les résultats de l'étude de la composition chimique des graines de $C$. mannii , A. hypogaea et $M$. oleifera sont présentés dans les tableaux 1 et 2 . Les valeurs moyennes des teneurs en protéines brutes, lipides totaux, glucides totaux, amidon total, humidité et cendres brutes sont présentées dans le tableau 1 , celles des teneurs en acides aminés dans le tableau 2. L'observation du tableau 1 révèle deux constituants majoritaires à savoir hypogaea, M. oleifera, sulfate d'aluminium, chlorure de fer III et de sulfate de fer II, à l'aide d'un spectrophotomètre DR890 à 525 nanomètres pour les ions $\mathrm{Al}^{3+}$ et à 510 nanomètres pour les ions $\mathrm{Fe}^{3+}$ et $\mathrm{Fe}^{2+}$.

Traitement Jar-Test: Le traitement Jar-Test, pour l'étude comparée des essais de clarification de l'échantillon d'eau brute de 94 NTU avec les solutions de C. mannii, A. hypogaea, M. oleifera et du sulfate d'aluminium a été effectué dans les conditions opératoires précédemment décrites

Teneur en matière organique apportée par les solutions de C. mannii, A. hypogaea et $M$. oleifera: La teneur en matières organiques a été évaluée par la méthode de l'oxydabilité au permanganate de potassium selon la norme EN ISO 8467 et par la détermination de la demande chimique en oxygène à l'aide d'un spectrophotomètre AL800 à 600 nanomètres.

Analyse statistique: Une analyse statistique a été effectuée sur les résultats des essais de clarification des échantillons d'eaux brutes de 128,6 NTU et 89,46 NTU, par le calcul d'écart type à l'aide du logiciel Microsoft Excel 2013.

les lipides et les protéines avec les teneurs respectives de $49 \mathrm{~g} / 100 \mathrm{~g}$ et de $37,40 \mathrm{~g} / 100 \mathrm{~g}$ pour C. mannii, de $48,40 \mathrm{~g} / 100 \mathrm{~g}$ et $30 \mathrm{~g} / 100 \mathrm{~g}$ pour $A$. hypogaea et de $38,90 \mathrm{~g} / 100 \mathrm{~g}$ et $36,50 \mathrm{~g} / 100 \mathrm{~g}$ pour M. oleifera. Ces résultats corroborent les données de la littérature qui indiquent que les lipides et les protéines sont les constituants majoritaires des graines de ces trois plantes (Prota, 2007).

Tableau 1 Teneurs des nutriments dans les graines de A. hypogaea, C. mannii et M. oleifera

\begin{tabular}{lccc}
\hline Paramètres $\mathbf{( g / 1 0 0 ~} \mathbf{g})$ & A. hypogaea & C. mannii & M. oleifera \\
\hline Protéines brutes & 30,0 & 37,4 & 36,5 \\
Lipides totaux & 48.4 & 49.0 & 38.9 \\
Glucides totaux & 16,4 & 5,7 & 17,3 \\
Amidon total & 5.9 & 2 & 12 \\
\hline Taux d'humidité & 3.1 & 3.9 & 3.9 \\
Teneur en cendres brutes & 2.2 & 4 & 3,5 \\
\hline
\end{tabular}

L'observation du tableau 2 montre que les deux acides aminés les plus abondants pour chaque plante, sont des acides aminés à chaînes latérales chargées à pH neutre. Des acides aminés à chaines latérales chargées positivement à pH neutre identifiés (Arginine, Lysine et Histidine), l'arginine est le plus abondant avec une teneur de $5510 \mathrm{mg} / 100 \mathrm{~g}$ dans C. mannii , 4850 $\mathrm{mg} / 100 \mathrm{~g}$ dans $M$. oleifera et $3550 \mathrm{mg} / 100 \mathrm{~g}$ dans $A$. 
hypogaea. Concernant les acides aminés à chaines latérales chargées négativement à pH neutre (Acide glutamique et Acide aspartique), l'acide glutamique est abondant dans les graines des trois plantes, avec les teneurs pratiquement égales dans les graines de $C$. mannii et de $M$. oleifera qui sont respectivement 6830 $\mathrm{mg} / 100 \mathrm{~g}$ et $6840 \mathrm{mg} / 100 \mathrm{~g}$; les graines de $A$. hypogaea ont présenté une teneur de $6300 \mathrm{mg} / 100 \mathrm{~g}$. L'acide aspartique est présent dans les graines de $A$. hypogaea avec une teneur de $6830 \mathrm{mg} / 100 \mathrm{~g}$.

Tableau 2. Teneurs en acides aminés dans les graines de A. hypogaea, C. mannii et M. oleifera

\begin{tabular}{|c|c|c|c|}
\hline $\begin{array}{l}\text { Acides aminés } \\
(\mathrm{mg} / 100 \mathrm{~g})\end{array}$ & A. hypogaea & C. mannii & M. oleifera \\
\hline \multicolumn{4}{|c|}{ Acides aminés à chaînes latérales chargées positivement à pH neutre (acides aminés basiques) } \\
\hline Histidine & 730 & 910 & 750 \\
\hline Lysine & 1040 & 1130 & 550 \\
\hline Arginine & 3550 & 5510 & 4850 \\
\hline \multicolumn{4}{|c|}{ Acides aminés à chaînes latérales chargées négativement à pH neutre (acides aminés acides) } \\
\hline Acide aspartique & 6830 & 3,14 & 1,4 \\
\hline Acide glutamique & 6300 & 6830 & 6840 \\
\hline \multicolumn{4}{|c|}{ Acides aminés à chaînes latérales non chargées à pH neutre, mais polaires } \\
\hline Sérine & 1500 & 1590 & 960 \\
\hline Tréonine & 750 & 1270 & 1370 \\
\hline Cysteine & 150 & 580 & 18,72 \\
\hline Tyrosine & 1330 & 1200 & 540 \\
\hline Asparagine & N.I. & N.I. & N.I. \\
\hline Glutamine & N.I. & N.I. & N.I \\
\hline \multicolumn{4}{|c|}{ Acides aminés à chaînes latérales non chargées à pH neutre et non polaires } \\
\hline Glycine & 1690 & 1860 & 1730 \\
\hline Alanine & 1230 & 1230 & 1350 \\
\hline Valine & 1330 & 1600 & 1300 \\
\hline Leucine & 2,05 & 2620 & 1,85 \\
\hline Isoleucine & 1070 & 1430 & 1060 \\
\hline Méthionine & 3630 & 850 & 650 \\
\hline Phénylalanine & 1680 & 1860 & 850 \\
\hline Tryptophane & 280 & 620 & 330 \\
\hline Proline & 1240 & 1330 & 1970 \\
\hline
\end{tabular}

Les résultats de l'étude sur les teneurs en acides aminés dans les graines de $C$. mannii sont similaires à ceux obtenus par Besong, où l'arginine $(4,07 \%)$ et l'acide glutamique $(4,51 \%)$ sont les acides aminés les plus abondants (Besong, 2011). Ibironke, a également observé des teneurs élevés en arginine $(9,193 \mathrm{~g} / 100 \mathrm{~g})$, en glutamate $(16,817 \mathrm{~g} / 100 \mathrm{~g})$, et en aspartate $(16,252$ $\mathrm{g} / 100 \mathrm{~g}$ ) dans les graines de C. mannii (Ibironke et Adegboye, 2013). Des teneurs élevées en acide glutamique $(25,65 \mathrm{~g} / \mathrm{kg})$ et en acide aspartique $(22,16$ $\mathrm{g} / \mathrm{kg}$ ) ont aussi été signalées par Zarkadas (Zarkadas, 1995) dans les feuilles de $M$. oleifera. La valeur moyenne de la teneur en lysine que nous avons observée dans les graines de $A$. hypogaea, est proche de celle citée dans la littérature $(926 \mathrm{mg} / 100 \mathrm{~g})$, (Prota,
2007). Les résultats de l'étude comparative de la composition chimique mettent en évidence des similitudes dans la composition chimique des graines de C. mannii. et $A$. hypogaea, d'une part et dans la composition chimique des graines de ces deux plantes avec celle des graines de M. oleifera d'autre part. Les similitudes des trois plantes pour la teneur en protéines et la composition en acides aminés à chaînes latérales chargées à pH neutre, justifient le choix qui a été porté sur les graines de $C$. mannii et de $A$. hypogaea, pour l'étude de l'activité coagulante de ces deux plantes.

Etude de l'activité coagulante

Essais de clarification des échantillons d'eaux brutes de 128,60 NTU et 89,46 NTU: Les résultats des essais de clarification des échantillons d'eaux 
brutes de 128,60 NTU et 89,46 NTU avec les solutions de poudre des graines de $A$. hypogaea et de C. mannii sont présentés respectivement dans les tableaux 3 et 4 . Pour chaque dose de solution liée aux trois traitements, les résultats des différents paramètres obtenus nous ont permis de calculer la moyenne. L'observation des valeurs moyennes des tableaux 3 et 4 , montre que l'addition de différentes doses des solutions des deux plantes a provoqué une diminution de la turbidité, pour chaque traitement qui a été effectué. La diminution de la turbidité est de 128,60 NTU à 3,73 NTU soit une réduction de $97 \%$ pour $A$. hypogaea. Cette diminution est de 89,45 NTU à 9,05 NTU soit une réduction de $90 \%$, pour C. mannii i. Après filtration des échantillons d'eaux traités et décantés, la turbidité de 3,73 NTU est passée à 0,24 NTU et celle de 9,05 NTU à 0,58 NTU. Ces valeurs des turbidités résiduelles sont conformes à la valeur de turbidité recommandée par l'OMS pour l'eau de boisson, soit 5 NTU. Ces résultats montrent l'élimination de la turbidité de l'eau par les solutions de poudre de graines de $C$. mannii et de $A$. hypogaea. Les solutions de poudre des graines des deux plantes ont provoqué la coagulation, qui est la neutralisation des particules colloidales responsables de la turbidité de l'eau. Ces résultats mettent donc en évidence l'activité coagulante des graines de C. mannii et de $A$. hypogaea dans la clarification des eaux de surface. Nous avons aussi noté une diminution des valeurs du $\mathrm{pH}$ de 5,75 à 4,80 pour $A$. hypogaea et de 6,45 à 5,94 pour $C$. mannii. La clarification de l'eau brute avec les solutions de $C$. mannii et de $A$. hypogaea nécessite un rectificateur d'acidité. Quant à la conductivité nous avons constaté une augmentation des valeurs de 10 $\mu \mathrm{S} / \mathrm{cm}$ à $227 \mu \mathrm{S} / \mathrm{cm}$ pour $A$. hypogaea et de $20 \mu \mathrm{S} / \mathrm{cm}$ à $300 \mu \mathrm{S} / \mathrm{cm}$, pour $C$. manniii. Ce résultat peut être expliqué par le fait que les solutions des deux plantes apportent des ions à l'eau clarifiée.

Tableau 3: Paramètres physico-chimiques pour l'eau brute de 128,60 NTU

\begin{tabular}{|c|c|c|c|c|c|c|}
\hline \multicolumn{3}{|c|}{$\begin{array}{l}\text { Paramètres } \\
\text { Physico-chimiques }\end{array}$} & $\begin{array}{c}\text { Température } \\
\left({ }^{\circ} \mathrm{C}\right)\end{array}$ & $\begin{array}{l}\text { Turbidité } \\
\text { (NTU) }\end{array}$ & $\mathrm{pH}$ & $\begin{array}{l}\text { Conductivité } \\
(\mu \mathrm{S} / \mathrm{cm})\end{array}$ \\
\hline \multicolumn{3}{|l|}{ Eau brute } & 27,7 & 128,60 & 5,75 & 10 \\
\hline $\mathbb{8}$ & & T1 & 28,30 & 4,42 & 4,84 & 140,1 \\
\hline ర্ঠ & 6000 & $\mathrm{~T} 2$ & 28,30 & 4,44 & 4,85 & 140 \\
\hline$\stackrel{8}{2}$ & 6000 & T3 & 28,30 & 4,44 & 4,83 & 140 \\
\hline 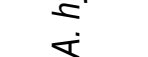 & & $M$ & 28,30 & 4,43 & 4,84 & 140 \\
\hline 0 & 7000 & $\mathrm{~T} 1$ & 28,22 & 3,72 & 4,96 & 160 \\
\hline 듣 & & $\mathrm{T} 2$ & 28,10 & 3,73 & 4,74 & 160 \\
\hline 윽 & & T3 & 28,20 & 3,74 & 4,71 & 160 \\
\hline 긍 & & $M$ & 28,17 & 3,73 & 4,80 & 160 \\
\hline 드 हᄐ & 8000 & T1 & 28,10 & 3,90 & 4,88 & 170 \\
\hline 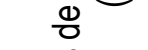 & & $\mathrm{T} 2$ & 28,00 & 3,92 & 4,64 & 170 \\
\hline$\Phi$ & & T3 & 28,03 & 3,90 & 4,60 & 170 \\
\hline ơ & & $M$ & 28,00 & 3,91 & 4,71 & 170 \\
\hline D & 9000 & $\mathrm{~T} 1$ & 28,00 & 5,00 & 5,4 & 190 \\
\hline 离 & & $\mathrm{T} 2$ & 28,10 & 5,02 & 5,43 & 190,1 \\
\hline 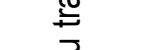 & & T3 & 28,40 & 4,99 & 5,49 & 190 \\
\hline Шَّ & & $\mathrm{M}$ & 28,17 & 5,00 & 5,44 & 190,03 \\
\hline
\end{tabular}

Analyse statistique: L'analyse statistique des résultats des essais de clarification des échantillons d'eaux brutes de 128,60 NTU et 89,45 NTU a montré pour $A$. hypogaea une dispersion de 0,01 à 0,02 pour la turbidité, de 0,01 à 0,15 pour le $\mathrm{pH}$ et de 0 à 0,06 pour la conductivité. Pour C. mannii., la dispersion est de 0,02 à 0,03 pour la turbidité, de 0,01 pour le $\mathrm{pH}$, et nulle pour la conductivité. 
Tableau 4: Paramètres physico-chimiques pour l'eau brute de 89,45 NTU

\begin{tabular}{|c|c|c|c|c|c|c|}
\hline \multirow{2}{*}{\multicolumn{3}{|c|}{$\begin{array}{l}\text { Paramètres } \\
\text { Physico-chimiques } \\
\text { Eau brute }\end{array}$}} & $\begin{array}{c}\text { Température } \\
\left({ }^{\circ} \mathrm{C}\right)\end{array}$ & $\begin{array}{c}\text { Turbidité } \\
\text { (NTU) }\end{array}$ & $\mathrm{pH}$ & $\begin{array}{l}\text { Conductivité } \\
(\mu \mathrm{S} / \mathrm{Cm})\end{array}$ \\
\hline & & & 28,10 & 89,45 & 6,45 & 20 \\
\hline \multirow{3}{*}{ 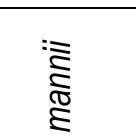 } & \multirow{3}{*}{6000} & $\mathrm{~T} 1$ & 28,50 & 9,85 & 5,96 & 110 \\
\hline & & $\mathrm{T} 2$ & 28,50 & 9,89 & 5,96 & 110 \\
\hline & & T3 & 28,40 & 9,86 & 5,97 & 110 \\
\hline ن & \multirow[b]{2}{*}{7000} & $M$ & 28,47 & 9,87 & 5,96 & 110 \\
\hline \multirow{8}{*}{ 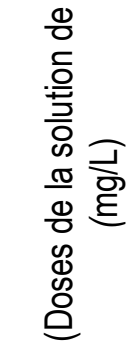 } & & $\mathrm{T} 1$ & 29,00 & 9,70 & 6,01 & 130 \\
\hline & & $\mathrm{T} 2$ & 29,00 & 9,69 & 6,03 & 130 \\
\hline & & T3 & 29,10 & 9,73 & 6,02 & 130 \\
\hline & \multirow{3}{*}{8000} & M & 29,03 & 9,71 & 6,02 & 130 \\
\hline & & $\mathrm{T} 1$ & 28,10 & 9,65 & 5,95 & 230 \\
\hline & & $\mathrm{T} 2$ & 28,90 & 9,66 & 5,94 & 230 \\
\hline & & T3 & 28,50 & 9,67 & 5,94 & 230 \\
\hline & \multirow{3}{*}{9000} & $M$ & 28,50 & 9,66 & 5,94 & 230 \\
\hline \multirow{2}{*}{ 兽 } & & $\mathrm{T} 1$ & 28,60 & 11,16 & 6,00 & 240 \\
\hline & & $\mathrm{T} 2$ & 28,90 & 11,19 & 5,82 & 240 \\
\hline \multirow{2}{*}{ 륨 } & & T3 & 28,50 & 11,14 & 5,99 & 240 \\
\hline & & $M$ & 28,67 & 11,16 & 5,94 & 240 \\
\hline
\end{tabular}

Etude comparée des essais de clarification de l'eau brute de 94 NTU

Dosage des ions $\mathrm{Al}^{3+}, \mathrm{Fe}^{3+}$ et $\mathrm{Fe}^{2+}$ dans les solutions préparées : La figure 1 présente les teneurs en ions $\mathrm{Al}^{3+}, \mathrm{Fe}^{3+}$ et $\mathrm{Fe}^{2+}$, dans les solutions préparées. La concentration en ion aluminium est de 0,12 $\mathrm{mg} / \mathrm{L}, 0,060$ $\mathrm{mg} / \mathrm{L}$ et $0,47 \mathrm{mg} / \mathrm{L}$ respectivement dans les solutions de
C. mannii , A. hypogaea et M. oleifera; alors qu'elle est de 765,02 $\mathrm{mg} / \mathrm{L}$ dans la solution de sulfate d'aluminium. Ces résultats montrent une très faible concentration en ion $\mathrm{Al}^{3+}$ dans les solutions des trois plantes; les teneurs en ions $\mathrm{Fe}^{2+}$ et $\mathrm{Fe}^{3+}$ dans ces plantes sont également faibles.

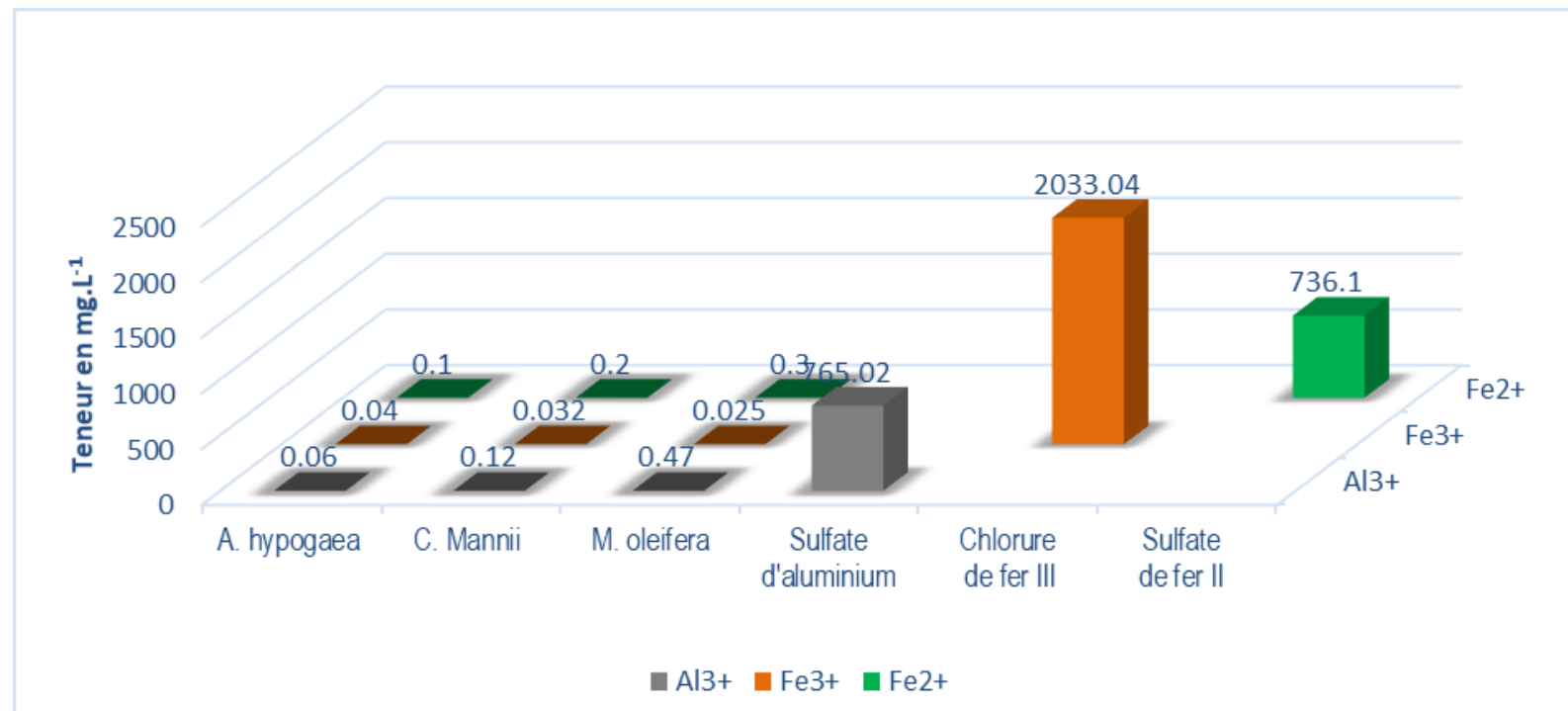

Figure 1 : Teneur en ions $\mathrm{Al}^{3+}$, $\mathrm{Fe}^{3+}$ et $\mathrm{Fe}^{2+}$ dans les solutions préparées 
Traitement Jar-Test: Les résultats des essais de clarification de l'eau brute de turbidité 94 NTU avec les solutions de C. mannii , A.hypogaea, M. oleifera et de sulfate d'aluminium sont présentés par les figures 2 à 4 . La figure 2 montre la variation de la turbidité en fonction des différentes doses des solutions coagulantes. La dose optimale pour chaque coagulant correspond au minimum de la courbe obtenue. Elle est de $16 \mathrm{mg} / \mathrm{L}$ pour le sulfate d'aluminium (Figure $2 \mathrm{c}$ ), de $1400 \mathrm{mg} / \mathrm{L}$ pour $M$. oleifera. (Figure $2 \mathrm{~b}$ ), de $7000 \mathrm{mg} / \mathrm{L}$ et de 9000 $\mathrm{mg} / \mathrm{L}$ respectivement pour $A$. hypogaea et $C$. mannii (Figure $2 \mathrm{a}$ ). Les turbidités résiduelles respectives sont de 1,79 NTU; 2,14 NTU; 13,55 NTU et 9,25 NTU. Après une filtration des échantillons d'eaux traités et décantés, les valeurs des turbidités résiduelles respectives sont passées à 0,11 NTU ; 0,14 NTU ; 0,87 NTU et 0,59 NTU. Ces résultats montrent l'intérêt des graines de $A$. hypogaea et de C. mannii dans la clarification des eaux de surface. En effet les valeurs de turbidité des eaux clarifiées par les graines des deux plantes sont proches de celles des eaux clarifiées par $M$. oleifera et le sulfate d'aluminium. Les figures 3 et 4 montrent une augmentation de la conductivité et une diminution du $\mathrm{pH}$ pour les quatre solutions coagulantes ; cette diminution du pH est moindre pour
M. oleifera. La diminution des valeurs du $\mathrm{pH}$ et l'augmentation de celles de la conductivité avaient été observé par Fatombi dans son étude comparative des essais de clarification des eaux de surface par les solutions de caséine acide, extraite de la crème de Cocos nucifera, de Moringa.oleifera et de sulfate d'aluminium (Fatombi, 2009).

Teneur en matière organique apportée par les solutions de C. mannii, A. hypogaea et M. oleifera: La figure 5 qui présente les résultats de la teneur en matière organique de l'eau brute de turbidité 94 NTU traitée avec les différentes solutions coagulantes, montre l'augmentation de l'indice de permanganate de 0 à $1,84 \mathrm{mg} \mathrm{L}^{-1} ; 4,32 \mathrm{mg} \mathrm{L}^{-1}$ et $5,36 \mathrm{mg} \mathrm{L}^{-1}$, respectivement pour l'eau brute traitée avec les solutions de M. oleifera, C. mannii, et $A$. hypogaea. Les valeurs de la demande chimique en oxygène augmentent de 26,4 à $43,2 \mathrm{mg} \mathrm{L}^{-1}$ pour l'eau brute traitée avec les solutions de $M$. oleifera et de $A$. hypogaea; puis à $45,2 \mathrm{mg} \mathrm{L}^{-1}$ pour celle traitée avec $C$. mannii . Ces résultats montrent que les solutions de $C$. mannii et de A. hypogaea, comme celle de M. oleifera apportent de la matière organique à l'eau traitée. 


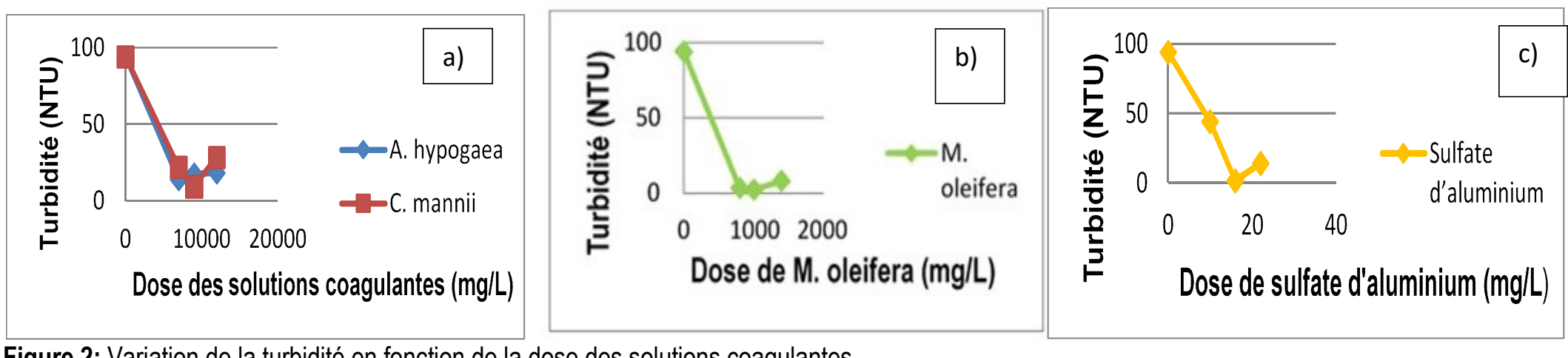

Figure 2: Variation de la turbidité en fonction de la dose des solutions coagulantes

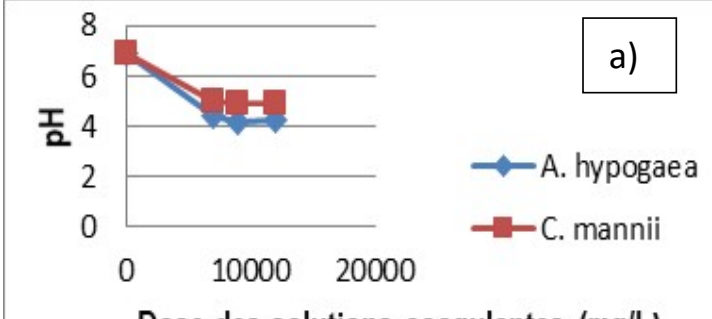

Dose des solutions coagulantes (mg/L)

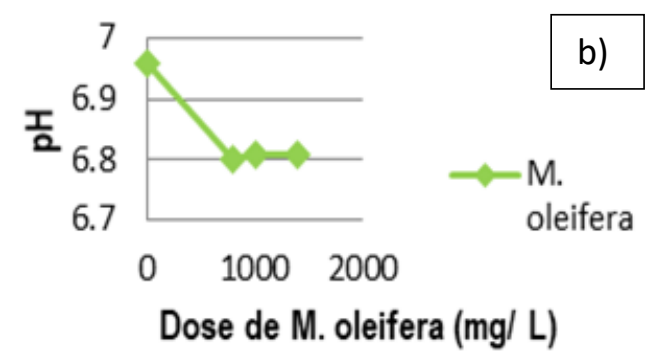

Dose de M. oleifera (mg/ L)

Figure 3: Variation du pH en fonction de la dose des solutions coagulantes

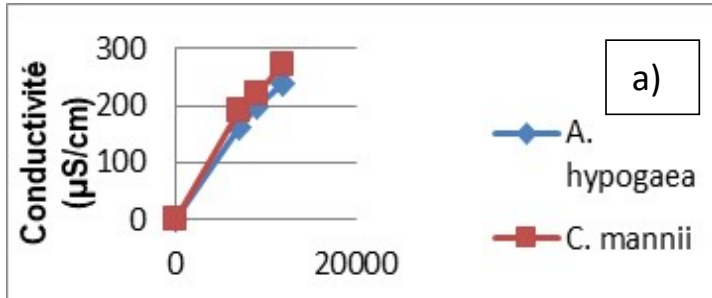

Dose des solutions coagulantes $(\mathrm{mg} / \mathrm{L})$

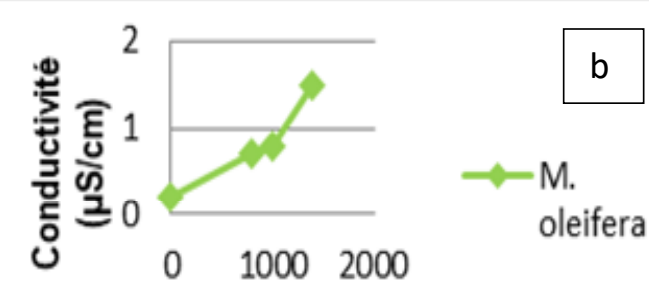

Dose de M. oleifera (mg/L)

Figure 4: Variation de la conductivité en fonction de la dose des solutions coagulantes

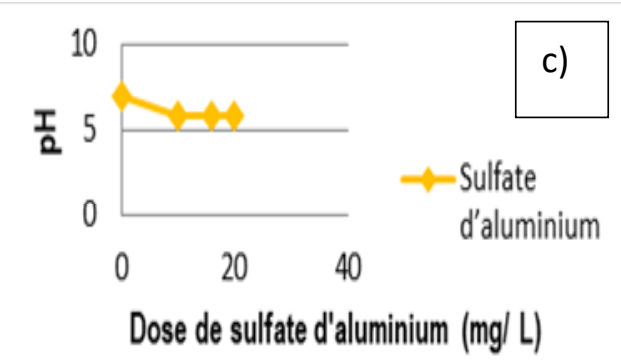




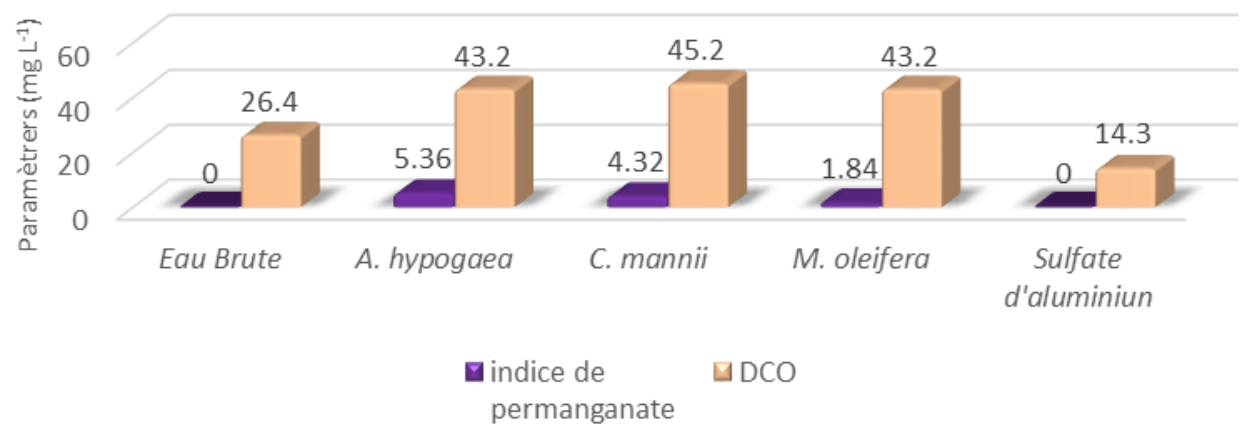

Figure 5 : Teneur en matière organique apportée à l'eau brute de 94 NTU traitée

\section{CONCLUSION}

Cette étude a montré que les graines de C. mannii, $A$. hypogaea et $M$. oleifera sont majoritairement constituées des protéines et des lipides; et leurs protéines ont des teneurs élevées d'acides aminés à chaînes latérales chargées à pH neutre. L'évaluation de l'activité coagulante par des essais de clarification de l'échantillon d'eau brute de 94 NTU a donné les doses optimales de $16 \mathrm{mg} / \mathrm{L}, 1400 \mathrm{mg} / \mathrm{L}, 7000 \mathrm{mg} / \mathrm{L}$ et 9000 $\mathrm{mg} / \mathrm{L}$ respectivement pour le sulfate d'aluminium, $M$.

\section{BIBLIOGRAPHIE}

Atakpama W., Kponor E. G. E., Kanda M., Dourma M., Nare M., Batawila K., Akpagana K., 2014. Moringa oleifera Lamarck: une ressource phytogénique à usage multiple. Rev. CAMES, 2(1), 6-14

Besong S. A., Ezekwe M.O., Fosung C.N. and Senwo Z.N., 2011. Evaluation of nutrient composition of African melon oilseed (Cucumeropsis manni naudin) for human nutrition. International journal of nutrition and metabolism, 3(8), 103108.

Binayke MS.R.A and Jadhav P.M.V., 2013. Application of natural coagulants in water purification. International Journal of Advanced Technology in Civil Engineering 2(1), $118-123$.

Cardot C., 2010. Les traitements de l'eau pour l'ingénieur. Procédés physico-Chimiques et biologiques. Ellipes Editions Marketing S.A. Paris, $302 \mathrm{P}$.

Degremont S., 2005. Memento technique de l'eau. Lavoisier SAS, 10 édition, Tomet 2,1718P.

Fatombi J.K., Josse R.G., Wotto V., Aminou T., Coulomb B., 2007. Paramètres physicochimiques de l'eau d'okpara traitée par les graines de Moringa oleifera. J. Soc. Ouest-Afr. Chim., 023, 75-79. oleifera, A. hypogaea et C. mannii . Des travaux ultérieurs visant à réduire la teneur en matière organique apportée à l'eau traitée seront effectué. Les résultats de cette étude mettent en évidence l'activité coagulante des graines des $C$. mannii et de $A$. hypogaea. Les graines de ces deux plantes peuvent être utilisées au niveau des ménages pour la clarification des eaux de surface, dans les régions rurales des pays en développement.

Fatombi J.K., Josse R.G., Mama D., Aminou T., 2009. Etude de l'activité floculante de la caséine acide extradite de la crème de Cocos nucifera dans la clarification des eaux de surface. Revue des Sciences de l'Eau, 22(1), 93-101

Hendricks D.W, 2006. Water Treatment Unit Processes: physical and chemical. CRC Press, $1266 \mathrm{P}$.

Ibironke A. et Adegboye S., 2013. Evaluation of Cucumeopsis mannii Seed Cake. Nature and Science, 11(7), 86-93.

Kabore A., Savadago B., Rosillon F., Straore A.F., Dianou D., 2013. Optimisation de l'efficacité des graines de Moringa Oleifera dans le traitement des eaux de consommation en Afrique Sub-saharienne: cas des eaux de Burkina Faso. Revue des sciences de l'eau, 26(3), 209-220.

Kweku Amagloh F. et Benang A., 2009. Effectiveness of Moringa oleifera seeds as coagulant for water purification. African Journal of Agricultural Research, 4(1), 119-123

Linangelo S.B., Kamango J. B., Mokili J. K. E., Monama T. O., Ulyel J. A. P, Kazada Z-A. M., 2018. Problématique d'accès à l'eau potable en milieu rural en RDC: cas de la ville urbano- 
rurale de Bumba. International Journal of Innovation and Scientific Research, 37(2), 130-138.

Lugube B., 2015. Production d'eau potable. Filières et procédés de traitement Dunod, Paris, $296 \mathrm{P}$

Nwaiwu N. E. et Lingmu B., 2011. Effect of settling time on turbidity removal using Moringa oleifera seed powder. Ozean Journal of Applied Sciences, 4(3), 195-208.

Ofoueme Berton Y., 2010. L'approvisionnement en eau des populations rurales au Congo Brazzaville. Les cahiers d'Outre-mer, $n^{\circ} 249,7-30$.

OMS, 2012. Objectifs du millénaire pour le développement. Aide-mémoire $n^{\circ} 290$

OMS, 2017. 2,1 milliards de personnes n'ont pas d'eau potable à domicile et plus du double ne disposent pas d'assainissement sûr. https: // www.who.int

PNUD, 2019. Eau propre et assainissement https: //www.undp.org

Prota, 2007. Ressources végétales de l'Afrique tropical 14. Oleagineux. Backhuys Publishers, Wageningen, Pays-Bas, 261P.

Sotheeswaran S., Nand V., Matakite M., 2011. Moringa Oleifera and other local seeds in water purification in developing countries. Research Journal of Chemistry and Environment 15(2), 135-138.

Zarkadas C.G., Yu Z.., Burrowis V.D., 1995. Protein quality of three new Canadian-developed naket oat cultivars using amino and compositional data. Journal of Agriculture and Food Chemistry, 43, 415 -421. 